\title{
Transcranial Laser Therapy of Post-Traumatic stress disorder in an Autistic Patient
}

\author{
Harold H Kraft* \\ Georgetown University Hospital, USA
}

Submission: January 02, 2019; Published: January 11, 2019

*Corresponding author: Harold H Kraft, Georgetown University Hospital, CMO at Laser MD Pain Relief, 1821 Wilshire Ave \#300, Santa Monica, CA 90403, USA

\begin{abstract}
A patient with severe chronic Post Traumatic Stress Disorder and severe Autism spectrum disorder was treated with Transcranial Laser Therapy. Initially large reductions in PTSD symptoms and small improvements in Autism were observed but were only partially durable. This is the first known treatment of comorbid Autism and PTSD with Transcranial Laser Therapy. Additionally, higher doses of laser over a shorter duration than previously reported were used.

Keywords: Autism spectrum disorder; Post-traumatic stress disorder; Near Infrared Laser; Photo biomodulation; Laser; Transcranial Laser Therapy; TLT; PTSD; ASD; NIL; LLLT

Abbreviations: ASD: Autism Spectrum Disorder; KJ: 1,000 Joules; PTSD: Post Traumatic Stress Disorder; TBI: Traumatic Brain Injury; TLT: Transcranial laser therapy; TX: Treatment
\end{abstract}

\section{Introduction}

Autism spectrum disorder (ASD) is a developmental disorder characterized by social communication impairments and restricted and repetitive behaviors [1]. Post-traumatic stress disorder (PTSD) is characterized by sleep disturbance, suicidal ideation, avoidance, and negative alterations in cognition and mood which occur in response to traumatic experiences. ASD prevalence in US children is estimated to be 1.5\% [2], and PTSD in US adults is estimated to be $3.6 \%$ [3].

Previous literature related to the comorbidity of PTSD and ASD is limited and includes examinations of comorbid occurrence rate [4-7], symptom overlap [8,9], ASD as a risk factor for PTSD [10], and a handful of case studies $[11,12]$. The goal of the current case study is to provide the first report of a novel therapy, Transcranial Laser Therapy (TLT), in treating a patient with comorbid ASD and PTSD.

TLT is a novel treatment of PTSD and Traumatic Brain Injury (TBI) which has shown very promising results in an initial report of ten patients [13], each receiving an average of 114,000 Joules. TLT uses Class IV, non-invasive, near-infrared lasers (NIL) with a frequency between 800 and 1,100nM which are FDA-cleared for treating pain. TLT is an off-label use of these lasers to penetrate the skull and induce photo biomodulation of the brain. Brain photo biomodulation is being studied in a wide variety of psychiatric and neurologic conditions, including PTSD, TBI, Alzheimer's, depression, and stroke [14]. No prior use of TLT in ASD has been reported. Additionally, this is the first report of a new "Manhattan Beach" protocol for TLT which uses 3 times higher doses than previously reported.

\section{Case Report}

\section{Clinic \& Protocols}

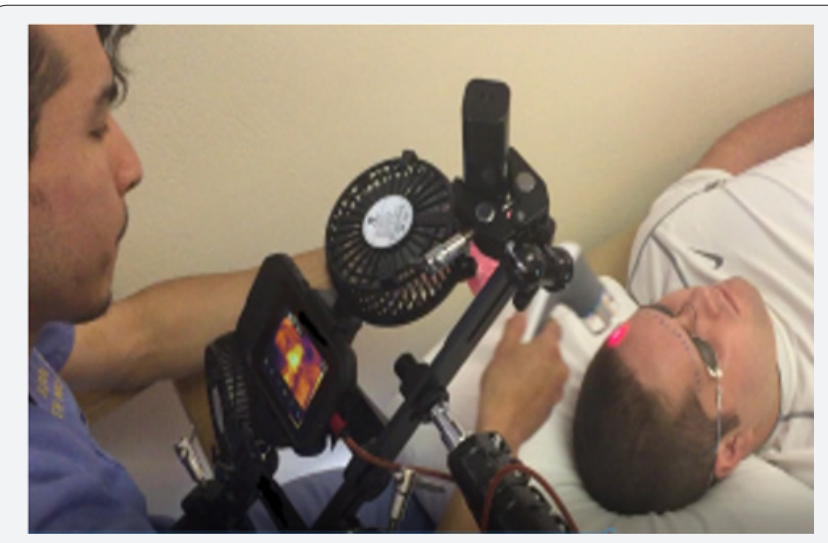

Figure1: Typical TLT, illustrating (left to right) laser operator, thermal imaging camera, cooling units, laser handpiece, laser aiming spot on forehead, supine patient with occlusive eye protection. 
This clinic specializes in high-dose, non-invasive, NIL treatments. The clinic primarily treats patients with advanced somatic and neuropathic pain, and in the past year has also treated 12 patients for PTSD and TBI with TLT. TLT is given with a $980 \mathrm{~nm}$ laser with a uniform density spot size of $3 \mathrm{~cm}$, with applied power in the 16-50watt range. Maximum skull skin temperature is continuously monitored with a thermal imaging camera producing 500 measurements per minute from $\sim 400$ points on the skull. Customized hardware provides 4-point forced-air cooling. Metal occlusive eyewear is worn by the patient during all TLT. See (Figure 1).

Our TLT regimen is a more aggressive modification of the "Denver" protocol used for TBI and PTSD in the initial report [13]. Our TLT "Manhattan Beach" protocol uses an average of 15 treatments every 2 days, each with 21,000 Joules, and totalling 300,000 Joules per patient. This is about 3 times faster and higher dosing than the previously reported protocol, as shown in Table 1. TLT is accompanied by a psychometric test battery before the treatment series, weekly during the treatment series, and after treatment conclusion at increasing intervals over 12 months.
Each battery of tests is self-completed by the patient online and includes measures of PTSD using the quantitative portion of the PCL-5, depression using both the Quick Inventory of Depressive Symptomatology SR-16 (QIDS) and the Beck Depression Index II (BDI), nightmares using the Disturbing Dreams and Nightmare Severity Index (DDNSI), and suicide using the Suicidal Behaviors Questionnaire-Revised (SBQ.) An internally developed questionnaire for side effects and qualitative patient comments was included in the battery.

Each treatment session begins with a brief free-form verbal assessment of nightmares and any TLT side effects. No cognitive behavioral therapy or any type of psychological support or counseling is provided. Next, the patient is placed supine on a comfortable, and treatment area are outlined with a washable marker. The typical treatment areas are shown in Figure 2. The thermal imaging camera and cooling units are placed and calibrated, and then the occlusive eye protection applied. Occlusion is tested by using the laser aiming light. The TLT is then applied using doses described in Table 1 . The total laser time is 3-7 minutes, and with the interlaced cooling periods, the patient is required to be moderately still for 5-20 minutes.

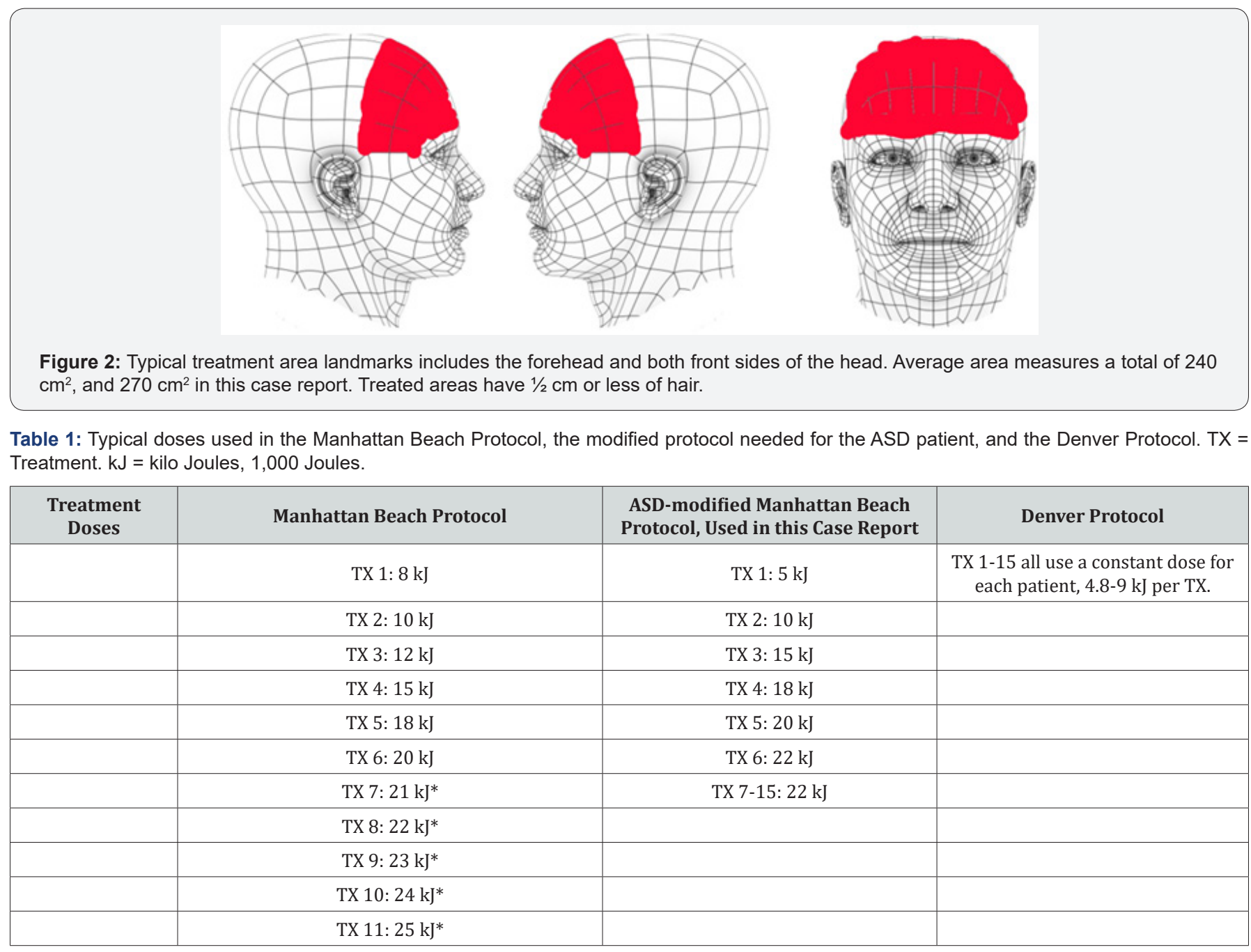




\section{Psychology and Behavioral Science International Journal}

\begin{tabular}{|c|c|c|c|}
\hline & TX $12+: 25$ kJ* & & \\
\hline Dose Notes & $\begin{array}{l}*=\text { Max dose is capped at } 20-25 \mathrm{~kJ} \text {. Laser } \\
\text { power in both the standard and ASD-modified } \\
\text { Manhattan Beach Protocols is } 16-50 \text { Watts, with } \\
\text { frequent cooling periods within each TX. }\end{array}$ & & \\
\hline $\begin{array}{l}\text { Total Length of } \\
\text { TX Series }\end{array}$ & $15-45$ days & 32 days & 60 days \\
\hline TX spacing & 11-30 TX, average 15 every 2 days. & 15 TX, average every 2 days. & 10-20 TX, average 15 every 4 days. \\
\hline \multirow[t]{2}{*}{$\begin{array}{l}\text { TX dose total \& } \\
\text { average }\end{array}$} & Total Dose $\sim 300 \mathrm{~kJ}$. & $\begin{array}{c}\text { Total Dose }=332 \mathrm{~kJ} . \text { Average Dose per } \\
\text { Treatment } \sim 23 \mathrm{KJ} .\end{array}$ & Total Dose 114 kJ. \\
\hline & Average Dose per Treatment $21 \mathrm{KJ}$. & & $\begin{array}{c}\text { Average Dose per Treatment } \sim 7 \\
\text { KJ. }\end{array}$ \\
\hline Treatment area & Frontal with bilateral temporal. $\sim 180-300 \mathrm{~cm}^{2}$. & $\begin{array}{l}\text { Frontal, with or without left or } \\
\text { bilateral temporal . } \sim 270 \mathrm{~cm}^{2} .\end{array}$ & $\begin{array}{l}\text { Frontal, with or without left or } \\
\text { bilateral temporal . } \sim 97-162 \mathrm{~cm}^{2} \text {. }\end{array}$ \\
\hline
\end{tabular}

\section{Presentation of Patient}

A 24-year-old male presented to the clinic for TLT treatment of PTSD. Patient was always accompanied by his mother who was the sole parent and had legal guardianship. The patient had been previously diagnosed with severe PTSD due to repeated sexual abuse and trauma starting at age 3 , as well as severe ASD. He was non-verbal and used a simple QWERTY tablet with fingerpointing for communication. His pointing was imprecise, and the mother often guessed entire sentences based on only a few fingerstrokes and his facial expression. He had a developmental age of 5-8 years old and brought books with a 5-6year reading level.
He was under the care of a psychotherapist weekly. The mother provided informed consent.

His PTSD symptoms included night terrors with screaming, severe non-communication and withdrawal, and rage accompanied by physically striking and injuring his mother. He had not smiled since age 3 , attributed to the repeated abuse. The mother administered the psychometric testing at home, with results shown in Table 2. PTSD score was severe, depression moderately severe, suicide score severe risk, and nightmare score severe.

Table 2: Patient's psychometric data.

\begin{tabular}{|c|c|c|c|c|c|c|}
\hline & $\begin{array}{c}\text { Cumulative Dose } \\
\text { kJ }\end{array}$ & PCL PTSD & BDI Depression & QIDS Depression & SBQ Suicide & DDNSI Nightmare \\
\hline \multirow[t]{2}{*}{ Test Day } & -- & Range $0-80$ & Range & Range & Range 3-18 & Range \\
\hline & & & $0-63$ & $0-45$ & & $0-40$ \\
\hline Pre-Treatment & & 72 & 33 & 30 & 15 & 33 \\
\hline $\begin{array}{c}\text { Day 15, after TX } \\
\# 7\end{array}$ & 134 & 66 & 30 & 28 & 15 & 24 \\
\hline $\begin{array}{c}\text { Day 23, after TX } \\
\quad \# 10\end{array}$ & 222 & 53 & 15 & 25 & 8 & 23 \\
\hline $\begin{array}{l}\text { Day } 34 \text {, post- } \\
\text { treatment day } 2\end{array}$ & 332 & 43 & 18 & 14 & 13 & $0^{*}$ \\
\hline $\begin{array}{l}\text { Day } 49 \text {, post- } \\
\text { treatment day } 17\end{array}$ & 332 & 59 & 16 & 19 & 15 & 6 \\
\hline
\end{tabular}

\section{Differential approach to patient due to ASD}

The patient's ASD presented multiple treatment and testing challenges. The patient would initially remove the eye protection and turn prone every 5 to 30 seconds, rather than the required 5 to 20 minutes. This improved over time, and by the fourth treatment he was able to lay supine for up to 5 minutes with frequent head movement and removed the eye protection no more than twice per treatment. By the seventh treatment, the patient was feeling substantially improved mood and therefore looking forward to each treatment. This presented a new challenge, as the patient would immediately, upon entering the room, lay supine for the treatment and start his internal clock, forcing us to use additional personnel to quickly prepare.
When asked prior to each treatment about side effects or nightmares, the patient would only communicate through his mother, limiting data collection. The biggest limitation was in psychometric testing. All the responses were subject to the mother's interpretation of the patient's finger-pointing on his tablet. Further, the length of time that the surveys required were well beyond the patient's focus limitations, which increased his frustration level and skewed the scores towards less favorable measures. Most critically, the tests themselves triggered PTSD symptoms including rage and striking his mother. Following the second post-treatment testing, the patient refused to participate in further testing. 


\section{Treatment and Results}

After first few treatments, the night terrors diminished. After seven treatments, the mother reported that the patient was "smiling for the first time in twenty years" at both her and acquaintances. He began to verbalize more words, and longer strings of words. During office visits, patient improved over time from nonverbal to occasionally verbal, and was strikingly more comfortable with staff, office equipment, and treatment positioning. Towards the end of the treatment series, he would frequently smile and even laugh during the treatment. The mother reports that the autistic symptom improvement during treatment nearly completely diminished within one-month post-treatment. Maximum improvements in psychometric test scores were PTSD $40 \%$, depression $55 \%$, suicide $47 \%$, and nightmares $82 \%$. After the full fifteen treatments and $332 \mathrm{~kJ}$ of TLT, improvements diminished to $18 \%$ for PTSD and $0 \%$ for suicide. Nightmares showed the most persistent decrease of $82 \%$.

\section{Discussion}

A case of severe PTSD in a severely autistic patient which responded to TLT is presented. In this case, substantial improvements in PTSD and ASD symptoms were observed during the treatment series but diminished or disappeared within a month following treatment completion. By comparison, of the 10 non-ASD adults treated in this clinic with TLT, 9 of 10 have had durable improvement in PTSD symptoms in the $30-100 \%$ range. Their psychometric scores usually continue to improve after treatment, and in one case a 95\% improvement has been observed to be durable for 12 months.

There are several possible reasons that this patient did not respond as well as the non-ASD adults treated with TLT:

1. Communication difficulty due to ASD via the mother's test interpretation contributed to less accurate scores.

2. Patient's PTSD triggers from the testing themselves contributed to worse results.

3. It could be that genetically the patient is simply not a responder to TLT.

4. The patient's particular type of PTSD, or severity of PTSD, may not be responsive to TLT.

5. ASD, alone or in combination with PTSD, may diminish TLT responses.

6. The patient's emotional immaturity may diminish TLT responses.

7. The TLT dose may be too low to get a durable impact, or the treatment frequency may be sub-optimal.
Future considerations for treating ASD with TLT should include a modification to the psychometric testing. The battery of tests should be spread out, rather than administered as a large group, and test frequency should probably be limited to every 2-3 weeks. Further adjustments to dose could be considered. The utility of TLT in ASD is yet to be defined.

\section{References}

1. American Psychiatric Association (2013) Diagnostic and Statistical Manual of Mental Disorders, ( $5^{\text {th }}$ edn.), American Psychiatric Publishing: Arlington, VA, USA.

2. Center for Disease Control and Prevention (2014) Prevalence of autism spectrum disorder among children aged 8 years-autism and developmental disabilities monitoring network, 11 sites, United States, 2010. Morbidity and Mortality Weekly Report. Surveillance Summaries 63: 1-21.

3. Post-Traumatic Stress Disorder (PTSD) (2018) National Institutes of Mental Health, United States.

4. van Steensel FJA, van Bögels SM, Perrin S (2011) Anxiety disorders in children and adolescents with autistic spectrum disorders: A metaanalysis. Clin Child Fam Psychol Rev 14(3): 302-317.

5. Mehtar M, Mukaddes NM (2011) Posttraumatic Stress Disorder in individuals with diagnosis of Autistic Spectrum Disorders. Res Autism Spectr Disord 5: 539-546.

6. De Bruin EI, Ferdinand RF, Meester S, de Nij PFA, Verheij F (2007) High rates of psychiatric comorbidity in PDD-NOS. J Autism Dev Disord 37(5): 877-886.

7. Storch EA, Sulkowski ML, Nadeau J, Lewin AB, Arnold EB, et al. (2013) The phenomenology and clinical correlates of suicidal thoughts and behaviors in youth with autism spectrum disorders. J Autism Develop Disord 43(10): 2450-2459.

8. Dell'Osso L, Dalle Luche R, Carmassi C (2015) A new perspective in post-traumatic stress disorder: Which role for unrecognized autism spectrum. International Journal of Emergency Mental Health and Human Resilience 17(2): 436-438.

9. Nietlisbach G, Maercker A (2009) Social cognition and interpersonal impairments in trauma survivors with PTSD. Journal of Aggression Maltreatment \& Trauma 18(4): 382-402.

10. Kerns CM, Newschaffer CJ, Berkowitz SJ (2015) Traumatic childhood events and autism spectrum disorder. J Autism Dev Disord 45(11): 3475-3486.

11. Stavropoulos K, Bolourian Y, Blacher J (2018) Differential Diagnosis of Autism Spectrum Disorder and Post Traumatic Stress Disorder: Two Clinical Cases. J Clin Med 7(4).

12. Trelles Thorne Mdel P, Khinda N, Coffey BJ (2015) Posttraumatic stress disorder in a child with autism spectrum disorder: Challenges in management. J Child Adolesc Psychopharmacol 25(6): 514-517.

13. Morries LD, Cassano P, Henderson TA (2015) Treatments for traumatic brain injury with emphasis on transcranial near-infrared laser phototherapy. Neuropsychiatr Dis Treat 11: 2159-2175.

14. Hamblin MR (2016) Shining light on the head: Photo biomodulation for brain disorders. BBA Clin 6: 113-124. 
Your next submission with Juniper Publishers will reach you the below assets

- Quality Editorial service

- Swift Peer Review

- Reprints availability

- E-prints Service

- Manuscript Podcast for convenient understanding

- Global attainment for your research

- Manuscript accessibility in different formats ( Pdf, E-pub, Full Text, Audio)

- Unceasing customer service

Track the below URL for one-step submission https://juniperpublishers.com/online-submission.php 